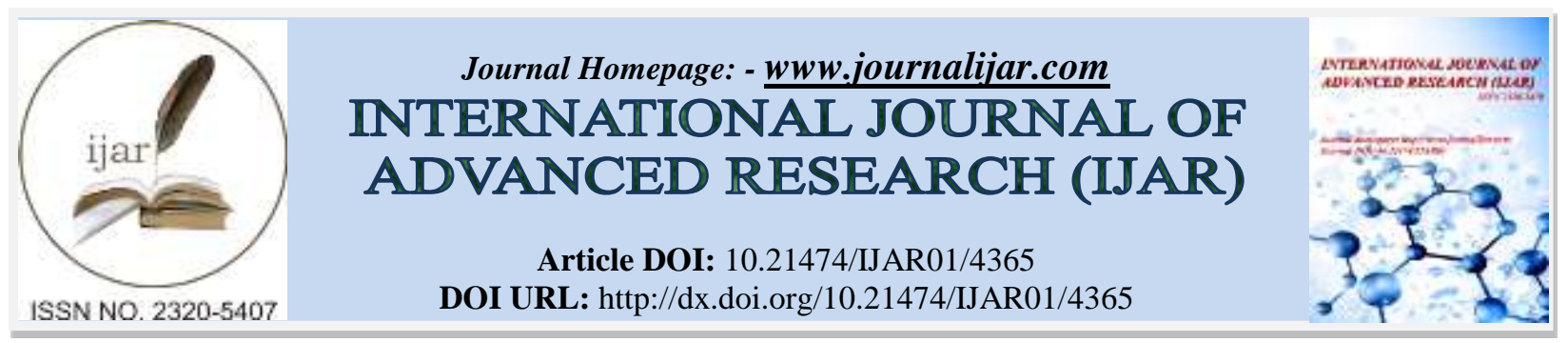

RESEARCH ARTICLE

\title{
IMPACT OF T315I MUTATION ON THE OUTCOME OF IMATINIB-RESISTANT CML IN EGYPTIAN PATIENTS
}

\author{
Mohamed Amr El-Menoufy*1, Amel Aly El Naggar ${ }^{2}$ and Laila El-Sayed Ziada ${ }^{3}$ \\ 1. Assistant Professor, Hematology Department, Medical Research Institute, Alexandria University. Egypt. \\ 2. Assistant Consultant, Hematology Department, Medical Research Institute, Alexandria University. Egypt. \\ 3. Professor, Hematology Department, Medical Research Institute, Alexandria University. Egypt.
}

\section{Manuscript Info}

\section{Manuscript History}

Received: 20 March 2017

Final Accepted: 16 April 2017

Published: May 2017

Key words:-

CML, imatinib resistance, T315I mutation

\section{Abstract}

Background: BCR-ABL T315I BCRT315I mutation is one of the most frequently identified mutations in Imatinib-resistant chronic myelogenous leukemia (CML) patients. However, its clinical impact remains controversial.

Objectives: to assess the clinical characteristics and outcome of T315I mutation in 79 Egyptian patients with CML after failure of Imatinib therapy.

Results: T315I mutation was present in 14 (18\%) patients. The level of resistance was heterogeneous. Forty nine patients were in chronic phase, 7 in accelerated phase, and 23 in blast crisis. Imatinib dose escalation in 2 resistant patients with T315+ showed transient good response. Responses were also observed in 3 out of 8 patients who were shifted to $2^{\text {nd }}$ generation Tyrosine Kinase Inhibitor (TKI) after Imatinib resistance. There was no difference in patient characteristics and survival between $\mathrm{T} 315 \mathrm{I}^{+}$and $\mathrm{T} 315 \mathrm{I}^{-}$patients.

Conclusion: the survival of $\mathrm{T} 315 \mathrm{I}^{+}$patients remains mainly dependent on the disease phase and the response duration. The difference in the disease outcome from most of previously reported studies may be related to mutation heterogeneity and to different ethnicity of patient population.

Evaluation of chromosomal abnormalities other than $\mathrm{Ph}^{1}$, and additional mutation at T315I identification is needed to know if there are other mechanisms of resistance.

Copy Right, IJAR, 2017, All rights reserved.

\section{Introduction:-}

The Philadelphia $\left(\mathrm{Ph}^{1}\right)$ chromosome is the hallmark of chronic myeloid leukemia (CML), it represents the fusion of the Abelson murine leukemia $(A B L)$ gene in chromosome 9 with the breakpoint cluster region $(B C R)$ gene in chromosome 22 . This fused $B C R-A B L$ oncogene encodes a deregulated, constitutively active tyrosine kinase that promotes growth, proliferation and apoptosis resistance.

Typically, CML has two or three phases: the disease is usually diagnosed in chronic phase (CP), followed by - with or without an acceleration phase (AP) - a blastic phase or crisis (BC). In the absence of specific treatment, the natural evolution of the disease is the progression to a fatal $\mathrm{BC} .^{(1)}$ 
Imatinib Mesylate (IM), the front-line treatment of CML is a selective tyrosine kinase inhibitor (TKI) that targets theadenosine triphosphate (ATP) binding site of the BCR-ABL protein, which results in the inhibition of phosphorylation of proteins involved in BCR-ABL signal transduction leading to growth arrest and apoptosis of the hematopoietic cells that express BCR-ABL without affecting the normal cells. ${ }^{(2)}$ Unfortunately, failure to respond to IM develops in some patients (primary resistance). In addition, approximately $15 \%$ of IM-treated patients, under the selective pressure of TKI, acquire recurrent BCR-ABL amino-acid exchanges ${ }^{(3)}$ which modify the conformation of IM binding site, preventing IM binding and result in decrease sensitivity of the drug, at different levels, and disease progression (acquired resistance). ${ }^{(4,5)}$

T315I BCR-ABL mutation remains one of the most frequently detected mutations in CML TKI-resistant patients. ${ }^{(6)}$ In patients bearing this mutation, IM resistance appears to be heterogeneous. It confers high levels of IM resistance in vitro, but different studies report a controversial clinical impact. Several studies suggested that the presence of a T315I mutation is associated poor survival. ${ }^{(7,8)}$ Conversely, other studies suggested no statistically significant survival difference between resistant patients with T315I mutation and those with other or no mutations. ${ }^{(9)}$ In addition, it is of crucial clinical importance to know whether IM dose escalation may - at least partially - overcome resistance and improve the disease outcome.

Although the importance of T315I mutation is increasing nowadays, published literature focusing on survival information for Egyptian patients with CML harboring this mutation remains very limited.

The aim of this study was to assess the clinical characteristics of Egyptian patients with CML harboring T315I mutation, and to evaluate their outcome after failure of IM therapy.

\section{Subjects:-}

The study included 79 CML Egyptian patients resistant to imatinib (IM) therapy between November 2010 and December 2016, regardless of the time of diagnosis and start of IM therapy.

All patients were in CP at diagnosis and had received the standard daily IM dose of $400 \mathrm{mg}$. Patients who had received allogeneic hematopoeitic stem cell transplantation were excluded from our study.

IM resistance was defined according European LeukemiaNet (ELN) guidelines ${ }^{(10)}$ as failure of achievement of either Complete Hematologic Response (CHR) after 3 months, Complete Cytogenetic Response (CCyR) at 12 months and Major Molecular Response (MMR) at 18 months of IM initiation (primary resistance), or loss of previously achieved hematologic, cytogenetic or molecular response (acquired resistance).

Among these 79 patients, 9 patients had failure to achieve CHR at 3 months and 23 patients had failure to achieve CCyR and/or MMR at 18 months; 10 patients had loss of CHR and CCyR and 7 patients had loss of CCyR; and 30 patients had progression to $\mathrm{AP} / \mathrm{BC}$.

An informed written consent was obtained from all participants prior to their inclusion in the study protocol, according to the ethical guidelines of the Medical Research Institute Alexandria University (Appendix1, Informed Written Consent for patient participation in a Clinical Research 2011)

\section{Statistical Analysis:-}

The chi-square test was used to determine the significance between variables. $P<0.05$ was considered statistically significant. Survival was analyzed according to Kaplan-Meier methods ${ }^{(11)}$ and Log rank test was used to compare between groups. Overall Survival (OS) was calculated from the time of Imatinib failure until date of death or last follow up. All statistical analyses were conducted with SPSS Version 24.0.Released 2016 (SPSS IBM Corp., Armonk, NY, USA), https://www 01.ibm.com/support/docview.wss?uid=swg21476197). 


\section{Methods:-}

T315I BCR-ABL gene Mutation was performed to all studied CML patients at the time of IM resistance, using allele specific oligonucleotide - polymerase chain reaction (ASO-PCR). ${ }^{(12)}$ Total RNA was extracted from whole blood using ABIOpure ${ }^{\mathrm{TM}}$ total RNA extraction kit (Alliance Bio, USA). Complementary DNA (cDNA) was generated by SuperScript III cDNA synthesis kit (Invitrogen, CA, USA) following the manufacturer's instructions. Sequence of forward and reverse primers for wild type ABL (WT), T315I mutant type (MT) and Internal control ( $\beta$-actin) used for ASO-PCR was adapted from previous report.[12] Sequences were specifically amplified in a PCR reaction. The amplified products were detected and assessed by electrophoresis on $2 \%$ ethidium bromide-stained agarose gel. The PCR products of T315I mutant, T315 wild type (WT) and $\beta$-actin gene were $158 \mathrm{bp}, 374 \mathrm{bp}$ and $540 \mathrm{bp}$ respectively (Figure 1).

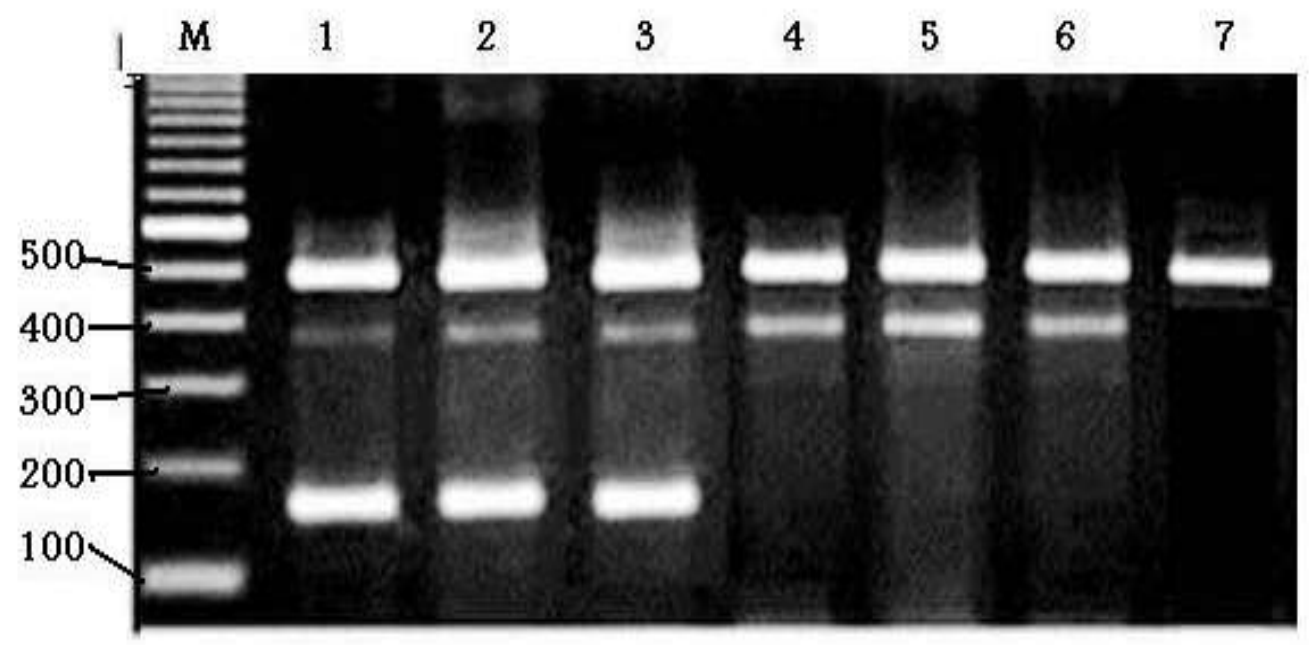

M: molecular size maker 100bp. Lanes 1-3 patients positive for T315I mutation. Lanes 4-6 patients negative for T315I mutation. Lane 7: internal control actin gene.

Figure 1:- Agarose gel electrophoresis of PCR products for detection of T315I mutation

\section{Results:-}

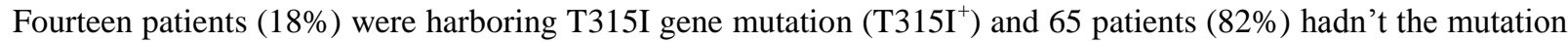
(T315I'), but they might harbor other mutation(s). The patients' characteristics at time of IM failure and the clinical outcome are summarized in Table [1]. There was no significant difference in characteristics between T315I+ and T315I- patients' groups.

The best Response before treatment Failure:-

Before treatment failure, $10(71 \%)$ patients with T315I mutation achieved CHR, CCyR and MMR while 2 patients (14\%) achieved CHR only. For T315I- patients, 37 (57\%) patients achieved CHR, CCyR and MMR; while 21 (32\%) patients achieved CHR only. There was no significant relationship between the mutation status (presence or absence of T315I) and CCyR to treatment ( $\mathrm{p}=0.244)$ [table 2].

Mutation status, response duration and the phase of the disease at Imatinib failure:-

Our study demonstrated no significant relationship between the mutation status and the phase of the disease at IM failure ( $p=0.828$ ) [table 3]. The Median duration of response (from start of treatment until treatment failure) in $\mathrm{T}_{315 \mathrm{I}^{+}}$and T315I- patients were 32 and 33.5 months respectively and no significant relationship was also found between the mutation status and duration of treatment response $(p=0.433)$ [table 4]. 
Table 1:- Characteristics of CML resistant patients at time of Imatinib failure and disease outcome .

\begin{tabular}{|c|c|c|c|}
\hline Characteristic & $\begin{array}{c}\text { T315I+ } \\
(n=14)\end{array}$ & $\begin{array}{c}\text { T315I- } \\
(n=65)\end{array}$ & $p$ value \\
\hline Patients, No. (\%) & $14(18 \%)$ & $65(82 \%)$ & \\
\hline Mean Age, years (range) & $48(33-65)$ & $46(25-69)$ & \\
\hline Male/Female & $6 / 8$ & $36 / 29$ & 0.143 \\
\hline $\begin{array}{cl}\text { Clinical features } \\
- & \text { Splenomegaly, no. }(\%) \\
- & \text { Fatigue, no. }(\%) \\
- & \text { Fever, no. }(\%) \\
- & \text { Bone pain, no. }(\%) \\
- & \text { Night sweats, no. }(\%) \\
- & \text { Bleeding, no. }(\%) \\
- & \text { Hepatomegaly, no. }(\%)\end{array}$ & $\begin{array}{l}12(86) \\
11(79) \\
06(43) \\
06(43) \\
07(50) \\
05(36) \\
03(21)\end{array}$ & $\begin{array}{l}63(97) \\
62(95) \\
35(54) \\
40(62) \\
36(55) \\
27(42) \\
23(35)\end{array}$ & $\begin{array}{l}0.847 \\
0.624 \\
0.847 \\
0.536 \\
0.392 \\
0.759 \\
0.662 \\
\end{array}$ \\
\hline $\begin{array}{cl}\text { Hematological parameters } \\
\text { - } & \text { Mean Hb level, g/dl (range) } \\
\text { - } & \text { Mean TLC x } 10^{9} / \mathrm{L} \text { (range) } \\
\text { - } & \text { Mean Basophil\% (range) } \\
\text { - } & \text { Mean Blast } \% \text { (range) } \\
\text { - } & \text { Mean Platelet count, } \times 10^{9} / \mathrm{L} \text { (range) }\end{array}$ & $\begin{array}{c}11.4(8.5-14.2) \\
52.9(5.9-139.5) \\
4.3(0.0-15.0) \\
11.7(1.0-43.0) \\
323.6(23.0-890.0)\end{array}$ & $\begin{array}{c}11.1(7.2-17.0) \\
28.8(4.0-105.0) \\
2.8(.0-12.0 \%) \\
11.8(0.0-90.0) \\
308.5(80.0-1254.0)\end{array}$ & $\begin{array}{l}0.781 \\
0.358 \\
0.366 \\
0.072 \\
0.777\end{array}$ \\
\hline $\begin{array}{cl}\text { Type of IM Resistance } \\
- & \text { Primary, No }(\%) \\
- & \text { Secondary, No }(\%)\end{array}$ & $\begin{array}{l}04(29) \\
10(71)\end{array}$ & $\begin{array}{l}28(43) \\
37(57)\end{array}$ & 0.162 \\
\hline $\begin{aligned} & \text { CML phase at Imatinib failure } \\
&- \text { Chronic, no. }(\%) \\
&- \text { Accelerated, no. }(\%) \\
&- \text { Blastic phase, no. }(\%)\end{aligned}$ & $\begin{array}{l}08(57) \\
01(07) \\
05(36)\end{array}$ & $\begin{array}{l}41(63) \\
06(09) \\
18(28)\end{array}$ & 0.828 \\
\hline Median response duration, months (range) & $32(12-62)$ & $33.5(3-70)$ & \\
\hline $\begin{array}{cl}\text { Disease Outcome } \\
- & \text { No. dead }(\%) \\
- & \text { No. alive }(\%)\end{array}$ & $\begin{array}{l}10(43) \\
04(57)\end{array}$ & $\begin{array}{l}29(35) \\
36(65)\end{array}$ & 0.063 \\
\hline
\end{tabular}

Abbreviations: $\mathrm{Hb}=$ Hemoglobin; $T L C=$ Total leucocyte count

Table 2:- Relation between Mutation status and Cytogenetic Response

\begin{tabular}{|c|c|c|c|c|c|c|c|}
\hline \multirow[t]{3}{*}{ Response achieved } & \multicolumn{4}{|c|}{ T315I Mutation status } & \multirow{2}{*}{\multicolumn{2}{|c|}{ Total $(n=79)$}} & \multirow[t]{3}{*}{$\boldsymbol{P}$} \\
\hline & \multicolumn{2}{|c|}{$\mathrm{T315I}^{+}(\mathrm{n}=14)$} & \multicolumn{2}{|c|}{ T315I $^{-}(n=65)$} & & & \\
\hline & No & $\%$ & No & $\%$ & No & $\%$ & \\
\hline CCyR at 12 months & 10 & 71 & 37 & 57 & 49 & 62 & $0.244 *$ \\
\hline
\end{tabular}

Abreviation: $C C y R=$ Complete Cytogenetic Response

*Non-significant

Table 3:- Relation between Mutation status and disease phase at time of imatinib failure

\begin{tabular}{|c|c|c|c|c|c|c|c|}
\hline \multirow[t]{3}{*}{ CML Phase } & \multicolumn{4}{|c|}{ T315I Mutation status } & \multirow{2}{*}{\multicolumn{2}{|c|}{ Total $(n=79)$}} & \multirow[t]{3}{*}{$P$} \\
\hline & \multicolumn{2}{|c|}{ T315I $^{+}(n=14)$} & \multicolumn{2}{|c|}{ T315I' $^{-}(n=65)$} & & & \\
\hline & No & $\%$ & No & $\%$ & No & $\%$ & \\
\hline Chronic Phase & 08 & 57 & 41 & 63 & 49 & 62 & $0.828 *$ \\
\hline Advanced stage & 06 & 43 & 24 & 37 & 30 & 38 & \\
\hline
\end{tabular}

*Non-significant 
Table 4:- Relation between Mutation and Duration of treatment response

\begin{tabular}{|c|c|c|c|c|c|c|c|}
\hline \multirow{3}{*}{$\begin{array}{l}\text { Duration of } \\
\text { response }\end{array}$} & \multicolumn{4}{|c|}{ T315I Mutation status } & \multirow{2}{*}{\multicolumn{2}{|c|}{ Total $(n=79)$}} & \multirow[t]{3}{*}{$\boldsymbol{P}$} \\
\hline & \multicolumn{2}{|c|}{$\mathrm{T315I}^{+}(\mathrm{n}=14)$} & \multicolumn{2}{|c|}{ T315I' $^{-}(n=65)$} & & & \\
\hline & No & $\%$ & No & $\%$ & No & $\%$ & \\
\hline$<1$ year & 02 & 14 & 06 & 09 & 08 & 10 & $0.828 *$ \\
\hline$>1$ year & 12 & 86 & 59 & 91 & 71 & 90 & \\
\hline
\end{tabular}

*Non-significant

Overall survival (OS):-

After a median follow-up of 42 months from IM failure, the estimate mean OS of all the studied CML patients with IM resistance was 42.8 months. Patients' OS from the time of IM failure according to the mutation status (presence or absence of T315I), phase of the disease at the time of IM failure, and duration of treatment response were shown in Figures $2 \mathrm{~A}$ through $\mathrm{C}$.

The estimate mean OS of T315I+ and T315I- patients were 38.2 and 43.7 months with 95\% CI of 28.6-47.9 and 38.648.8 months respectively (figure 2-A) with no significant difference in survival between the 2 groups of patients $(\mathrm{p}=0.111)$. Thirty nine of 79 patients $(49.4 \%)$ died, including 11 of $49(22.4 \%)$ patients in CP, 5 of $7(71.4 \%)$ patients in $\mathrm{AP}$, and all 23 patients $(100 \%)$ in $\mathrm{BC}$. In CP, AP and BC, the estimate mean OS from IM failure were 56.9, 32.7 and 15.7 months respectively with highly significant difference $(p<0.001)$. Advanced disease phase $(\mathrm{AP}$ and $\mathrm{BC})$ is significantly associated with poor survival (figure 2-B).

The estimate mean OS for patients with treatment response duration less than one year and $\geq 1$ years were 31.1 and 44.1 months with $95 \%$ CI of 22.0-40.3 and 39.3-49.0 months respectively with significant difference $(p=0.04)$, short IM response duration is associated with poor survival (figure 2-C).

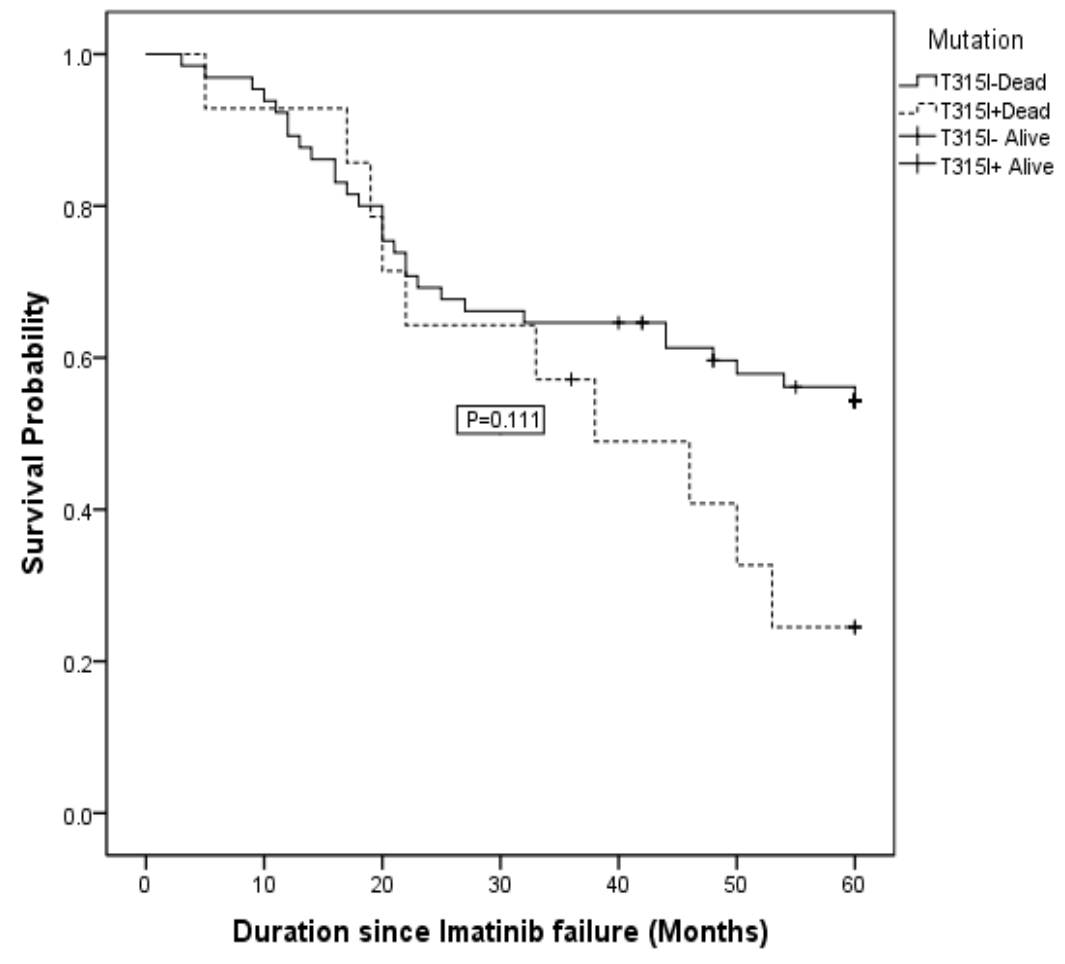

Figure 2-A:- Survival of resistant patients since IM failure according to the mutation status. 


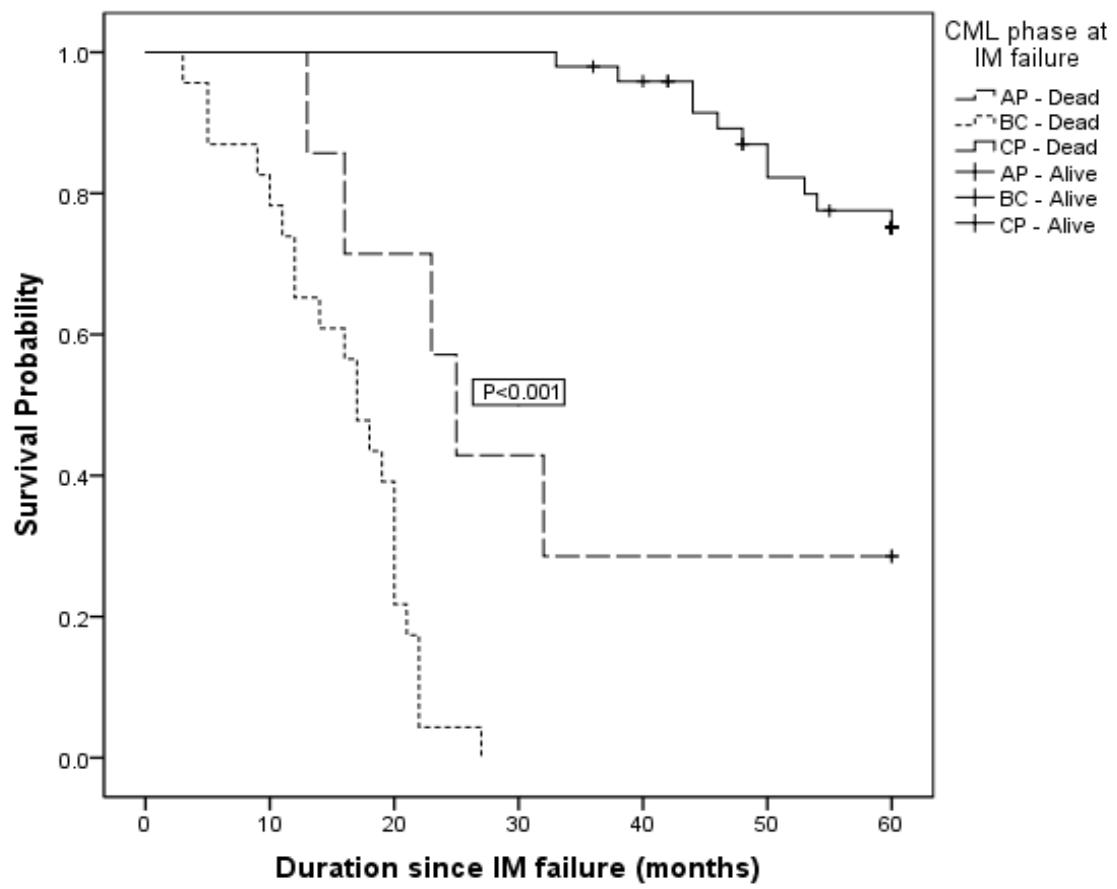

Figure 2-B:- Survival of resistant patients since IM failure according to the phase of the disease at IM failure

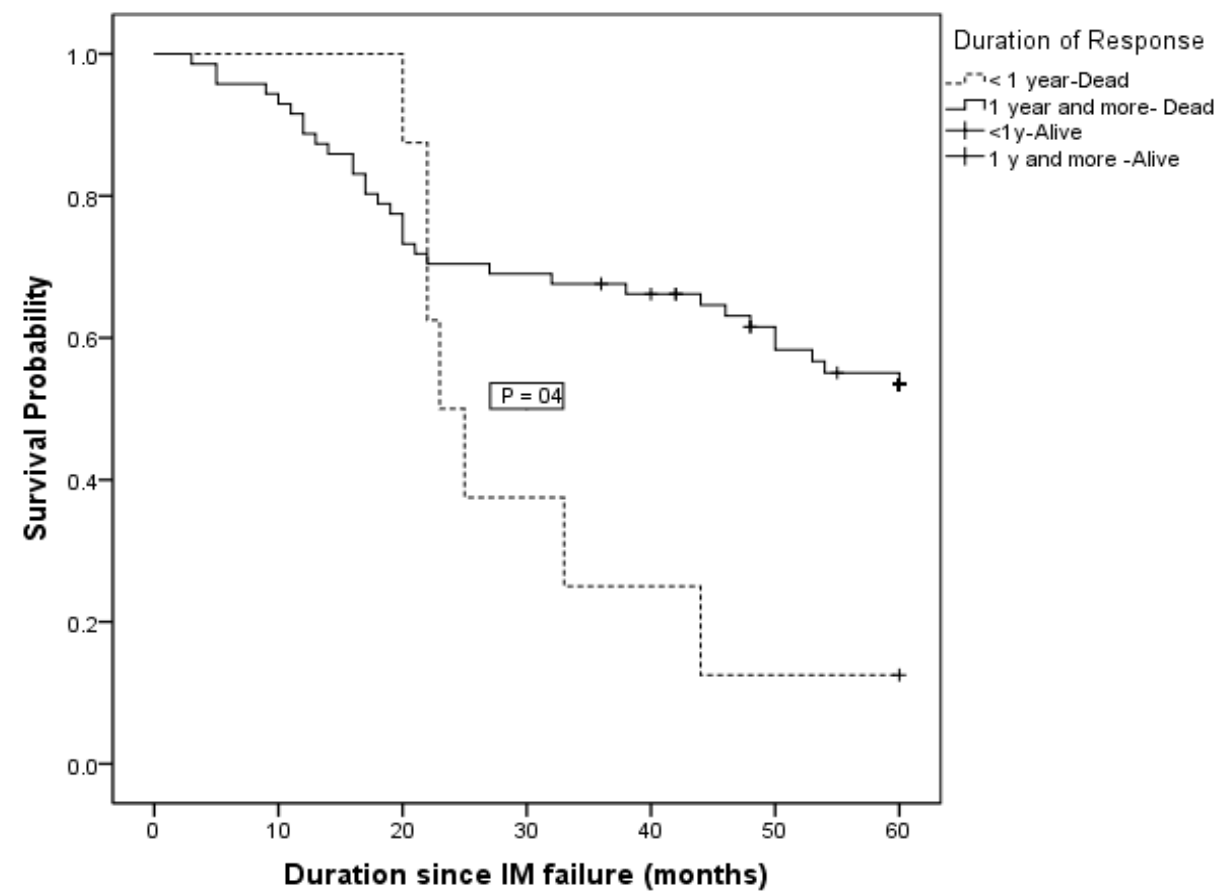

Figure 2-C:- Survival of resistant patients since IM failure according to the duration of treatment response. 


\section{Discussion:- \\ Frequency of T315 Mutation:-}

We present our experience for T315I mutation among 79 CML Egyptian patients who failed IM therapy and we identified the mutation in 14 patients $(18 \%)$.

The reported T315I mutation frequency in IM-resistant CML patients ranged between $2 \%$ and $20 \% .^{(13-16)}$ This variability may be related to sensitivities of the technical methods used to detect the mutation ${ }^{(9,16)}$, the time point of analysis, the treatment response ${ }^{(15)}$, the proportion of patients in primary versus secondary resistance in the different studies. In agreement with previous studies ${ }^{(17,18)}$, more patients with secondary resistance $(10 / 47,21.3 \%)$ developed T315I mutation during IM treatment than those with primary resistance $(4 / 32,12.5 \%)$.

\section{The best cytogenetic response to IM:-}

Cytogenetic response is the gold standard for assessing optimal response and predicting long-term outcome as the primary goal of CML therapy is still the achievement of CCyR.

Ten out of 14 patients with T315I mutation showed an optimal cytogenetic response to IM therapy immediately before the occurrence of resistance or progression and, in concordance with the finding of Norozi et al ${ }^{(15)}$, there was no significant relationship between the mutation status (presence or absence of T315I) and CCyR to treatment.

\section{Time of IM resistance and mutation detection from treatment initiation:-}

In our study, most of the T315I+ patients $(12 / 14,86 \%)$ developed IM resistance after one year of treatment. However, the onset of T315I mutations during IM treatment of CML remains challenging. Some authors ${ }^{(19,20)}$ suggested that mutations may be present at diagnosis prior to therapy, while others ${ }^{(21)}$ postulated that no instances of mutations develop in early CP before IM initiation and most will develop during treatment. Thus, the presence of T315I mutation remains a time-dependent covariate.

\section{Mutation status and Disease phase at time of IM failure:-}

In our study, there was no significant relationship between the mutation status and the phase of the disease at IM failure. This observation might partly be explained by the emerge of additional chromosomal and/or genomic alterations frequently occurred at the time of progression in advanced phase CML in patients with and without T315I mutation as already postulated by different authors. ${ }^{(22,23)}$

Among the T315I+ patients, all the 5 patients in $\mathrm{BC}$ died and one patient in AP was still alive. This patient developed a sustained CCyR cytogenetic response on second generation TKI. This may support the finding of Jabbour et al. ${ }^{(9)}$ Eight patients were in CP, suggesting that this mutation is not restrictly confined to patients in advanced stage disease.

On the other hand, 23 out of 24 resistant patients with undetectable T315 mutation in advanced stage (18 patients in $\mathrm{BC}$ and 5 patients in AP) died. Therefore, the occurrence of IM resistance and disease progression in T315I negative group of patients may imply the presence of other BCR-ABL mutations (non-investigated) or additional genetic abnormalities affecting other unknown BCR-ABL independent mechanisms of IM resistance/progression that provides the mutated clone with a proliferative advantage. ${ }^{(24-27)}$

Recently, $\mathrm{Ng}$ and associates ${ }^{(28)}$ identified a novel mechanism of primary resistance to TKIs among East Asians compared with other ethnic groups. The TKI resistance was secondary to a germline deletion polymorphism of the BH3 domain of BCL2-like 11 (BIM) that is required for TKIs to induce apoptosis in cancer driven by kinases.

It was noticed that 2 patients in advanced phase (AP) (one patient with and the other without T315I mutation) were escaped from death. Interestingly, these 2 patients received Interferon- $\alpha$ (IFN $\alpha$ ) either before IM treatment or after development of resistance. It remains unclear whether IFN- $\alpha$ may induce a protective effect for patients treated with IM ${ }^{(14)}$, or exerts a specific activity on the T315I mutated clone ${ }^{(29)}$ or whether it is simply the withdrawal of the TKI, that temporarily improves the outcome of these 2 patients. ${ }^{(30)}$ However, Jabbour et al ${ }^{(13)}$ suggested that there is a correlation between prior IFN therapy and the occurrence of mutations. Thus, the benefit of IFN- $\alpha$ use either before or combining with IM or after development of resistance (as salvage therapy) deserves profound study. 
Thirty nine of 79 (49\%) resistant patients in CP were still alive due to IM dose escalation or shift to 2nd generation TKI, as early intervention that prevented progression of the disease.

\section{Response to IM dose escalation and second TKI after IM failure:-}

In order to overcome resistance, dose escalation of IM (600-800 mg/day) was attempted in 44/79 (56\%) of resistant

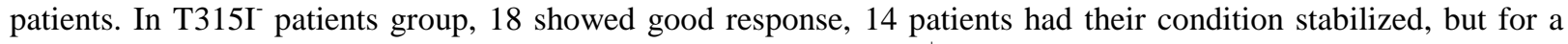
shorter duration and 6 patients showed no improvement. For T315I ${ }^{+}$patients, 4 patients in advanced stage showed inadequate response and 2 patients in CP showed good response during 11 and 32 months respectively. Thus, unlike previous studies ${ }^{(31,32)}$, our study showed that IM dose escalation in patients with T315I mutation could yield, although not sustained, a good response. The good response to dose escalation may support the suggestion of Yamamoto et al ${ }^{(33)}$ that T315I mutation develops IM resistance through increasing the ABL tyrosine kinase activity and dose escalation might overcome this increased activity. Ernst et al ${ }^{(34)}$ also reported a good response to therapy with $600 \mathrm{mg}$ IM during 37 months in a CP-CML patient harboring T315I mutation. Thus, these findings are increasingly supporting the suggestion of Nicolini et al ${ }^{(14)}$ that the levels of IM resistance, regardless of the presence or absence of T315I mutation, are heterogeneous, ranging from slightly reduced sensitivity to IM (overcome by IM dose escalation) to absolute insensitivity to IM (requiring other treatment modality).

Among the 14 patients with T315I, 8 patients were shifted to second generation TKI (Dasatinib) after failure of IM treatment. Responses were observed in 3 patients (Sustained CHR, sustained CHR/CCyR, sustained PHR/transient minor CyR). This finding did not match with most of previous studies, ${ }^{(35-38)}$ which showed that resistant CML patients bearing T315I mutation do not respond to second generation TKI, our finding in this respect may support that of Jabbour et al ${ }^{(9)}$ who first reported sustained responses of T315I-bearing CML clones to second TKI, indicating that this mutation does not predict for resistance in all cases.

The presence of controversy in the responses to IM dose escalation and $2^{\text {nd }}$ generation TKI in some of our T315I+ IM-resistant patients from most of the previous studies might be - at least partially- related to the mutation pattern heterogeneity and to the geographical and ethnical differences of the studied population as suggested by $\mathrm{Ng}$ et al ${ }^{(28)}$ and Nicolini et al ${ }^{(39)}$ in their study which included patients from 9 countries (from Europe, Asia and North America).

\section{Impact of mutational status, disease phase and response duration on OS:-}

Although in vitro studies ${ }^{(40)}$ demonstrate that the BCR-ABL T315I mutation provides the mutated clone with a proliferative capacity over BCR-ABL WT cells and favors disease transformation, our study showed no difference in survival between patients with, and without T315I, in concordance with the previous studies of Jabbour et al ${ }^{(9,13)}$ who concluded that neither the presence of mutations nor the type of mutation was associated with survival among patients who failed IM. On the contrary, other studies ${ }^{(14,35,41)}$ suggested that the presence of T315I mutation is associated with significantly poor prognosis and reduced overall survival. Yet, it remains unclear whether the presence of T315I mutation is actually responsible for disease progression through disruption of IM binding or interacts with other general factors of progression such as cytogenetic clonal evolution or additional mutations or it is simply present in a cell that is resistant to IM for other unknown reasons. Therefore, chromosomal abnormalities other than the $\mathrm{Ph}^{1}$, and additional mutation at T315I identification should be evaluated to know if there are other mechanisms of resistance. Thus, the impact of T315I mutation on survival depends on several factors. ${ }^{(13)}$ In addition, the extent of resistance of IM and other TKIs to T315I mutation may vary greatly ${ }^{(42)}$ and it remains unclear why some patients developed IM-resistance with detectable levels of T315I clones while others do not. ${ }^{(43)}$

Our study demonstrated also that survival is mostly dependent on the duration of IM response and the disease phase at the time of IM failure. Shorter response duration and advanced stage disease were associated with poor survival.

On the other hand, the above results indicated no significant relationship between T315I mutation, duration of response to treatment, $\mathrm{CCyR}$ to treatment, blood parameters and patients' clinical signs. Therefore, the evaluation of this mutation is recommended in all the patients in different phases of disease, even with a favorable response to therapy, which may be associated with relapse of their disease in the future and requires comprehensive studies in a longer period of time. ${ }^{(15)}$ 


\section{Conclusion:-}

Therefore, it had be concluded that the detection of T315T mutation in BCR-ABL-positive Egyptian resistant patients has nothing to do with patient survival, treatment period and CCyR and the survival of these patients remains largely dependent on the disease phase at the time IM failure, as well as the duration of response to IM therapy.

Lastly, the fact that we did not observe clear treatment pattern after T315I mutation detection highlights the need for evaluation of chromosomal abnormalities other than the $\mathrm{Ph}^{1}$, and additional mutation at T315I identification to know if there are other mechanisms of resistance.

\section{Conflict of Interest:-}

The authors declare no conflicts of interest in preparing this article.

\section{References:-}

1. Jabbour E, Kantarjian H. Introduction: chronic myelogenous leukemia (CML). Semin Hematol 2007; 44:S1-3.

2. Deininger M, Goldman, Lydon N, Melo J. The tyrosine kinase inhibitor CGP57148B selectively inhibits the growth of BCR-ABL-positive cells. Blood 1997; 90: 3691-3698.

3. Shah NP, Nicoll JM, Nagar B, Gorre ME, Paquette RL, Kuriyan J, et al. Multiple BCRABL kinase domain mutations confer polyclonal resistance to the tyrosine kinase inhibitor imatinib (STI571) in chronic phase and blast crisis chronic myeloid leukemia. Cancer Cell. 2002;2(2):117-25.

4. Quintás-Cardama A, Kantarjian HM, Cortes JE. Mechanisms of primary and secondary resistance to imatinib in chronic myeloid leukemia. Cancer control 2009; 16(2): 122-3.

5. Luzzatto L, Melo JV. Acquired resistance to imatinib mesylate: Selection for pre- existing mutant cells. Blood $2002 ; 99(9): 3472-5$.

6. Apperley JF. Part I: mechanisms of resistance to imatinib in chronic myeloid leukaemia. Lancet Oncol. 2007;8(11):1018-29.

7. Branford S, Rudzki Z, Walsh S, Parkinson I, Grigg A, Szer J, et al. Detection of $B C R-A B L$ mutations in patients with CML treated with imatinib is virtually always accompanied by clinical resistance, and mutations in the ATP phosphate-binding loop (P-loop) are associated with a poor prognosis, Blood 2003; 102:276-283.

8. Soverini S, Martinelli G, Rosti G, Bassi S, Amabile M, Poerio A, et al. ABL Mutations in Late Chronic Phase Chronic Myeloid Leukemia Patients With Up-Front Cytogenetic Resistance to Imatinib Are Associated With a Greater Likelihood of Progression to Blast Crisis and Shorter Survival: A Study by the GIMEMA Working Party on Chronic Myeloid Leukemia. J Clin Oncol 2005; 18: 4100-4109.

9. Jabbour E, Kantarjian H, Jones D, Breeden M, Garcia-Manero G, O'Brien S, et al. Characteristics and outcomes of patients with chronic myeloid leukemia and T315I mutation following failure of imatinib mesylate therapy. Blood 2008;112: 53-55.

10. Baccarani M, Deininger MW, Rosti G, Hochhaus A, Soverini S, Apperley JF, et al. European LeukemiaNet recommendations for the management of chronic myeloid leukemia: 2013. Blood 2013; 122(6):872-884.

11. Kaplan EL, Meier P. Non-parametric estimation from incomplete observation. J Am Stat Assoc 1985: 457-481.

12. Wongboonma W, Thanegnoppakyhum W, Auewarakul CU. A single- tube allele specific -polymerase chain reaction to detect T315I resistant mutation in chronic myeloid leukemia patients. J Hematol 2011; 4: 7- 11.

13. Jabbour E, Kantarjian H, Jones D, Talpaz M, Bekele N, O'Brien S, et al. Frequency and clinical significance of BCR-ABL mutations in patients with chronic myeloid leukemia treated with imatinib mesylate. Leukemia. 2006; 20(10):1767-1773.

14. Nicolini FE, Corm S, Le Q-H, Sorel N, Hayette S, Bories D, et al. Mutation status and clinical outcome of 89 imatinib mesylate resistant chronic myelogenous leukemia patients: a retrospective analysis from the French intergroup of CML (Fi-LMC GROUP). Leukemia 2006; 20(6):1061-1066.

15. Norozi F, Mohammadi-asl J, Vosoughi T, Jalali Far MA, Malehi AS, Saki N. Incidence of T315I mutation in BCR/ABL-positive CML and ALL patients. Front Biol 2016, 11(5): 404-411.

16. Kagita S, Uppalapati S, Jiwatani S, Linga V G, Gundeti S, Nagesh N, Digumarti R. Incidence of Bcr-Abl kinase domain mutations in imatinib refractory chronic myeloid leukemia patients from South India. Tumour Biol 2014; 35(7): 7187-7193.

17. Soverinia S, Branford S, Nicolini FE, Talpazf M, Deiningerg MWN, Martinellia G, et al. Implications of BCRABL1 kinase domain-mediated resistance in chronic myeloid leukemia. Leuk Res 2014; 38:10-20. 
18. Meggyesi N, Kozma A, Halm G, Nahajevszky S,$\underline{B a ́ t a i ~ A}$, Fekete $\mathrm{S}$, et al. Additional chromosome abnormalities, BCR-ABL tyrosine kinase domain mutations and clinical outcome in Hungarian tyrosine kinase inhibitor-resistant chronic myelogenous leukemia patients. Acta Haematol 2012; 127: 34-42.

19. Roche-Lestienne C, Soenen-Cornu V, Grardel-Duflos N, Lai JL, Philippe N, Facon T et al. Several types of mutations of the Abl gene can be found in chronic myeloid leukemia patients resistant to STI 571, and they can pre-exist to the onset of treatment. Blood 2002; 100: 1014-1018.

20. Kreuzer KA, Le Coutre P, Landt O, Na IK, Schwarz M, Schultheis K et al. Preexistence and evolution of imatinib mesylate-resistant clones in chronic myelogenous leukemia detected by a PNA-based PCR clamping technique. Ann Hematol 2003; 82: 284-289.

21. Willis S, Lange T, Demehri S, Otto S, Crossman L, Niederwieser D, et al. High sensitivity detection of BCRABL kinase domain mutations in imatinib-naive patients: correlation with clonal cytogenetic evolution but not response to therapy. Blood 2005; 106(6):2128-37.

22. Hochhaus A, Kreil S, Corbin AS, La Rosée P, Muller MC, Lahaye T, et al. Molecular and chromosomal mechanisms of resistance toimatinib (STI571) therapy. Leukemia 2002; 16: 2190-2196.

23. Al-Ali HK, Heinrich MC, Lange T, Krahl R, Mueller M, Müller C, et al. High incidence of BCR-ABL kinase domain mutations and absence of mutations of the PDGFR and KIT activation loops in CML patients with secondary resistance to imatinib. Hematol J 2004; 5: 55-60.

24. Hochhaus A. Dynamics of mutated clones in chronic myeloid leukemia patients. Haematologica 2009;78-82.

25. Bixby D, Talpaz, M. Mechanisms of resistance to tyrosine kinase inhibitors in chronic myeloid leukemia and recent therapeutic strategies to overcome resistance. Hematology Am Soc Hematol Educ Program 2009: 461476

26. Jabbour E, Kantarjian H, Cortes J. Chronic myeloid leukemia. Emerg Cancer Ther 2011b; 2: 239-258.

27. Zhang WW, Cortes JE, Yao H, Zhang L, Reddy N, Jabbour E, et al. Predictors of primary imatinib resistance in chronic myelogenous leukemia are distinct from those in secondary imatinib resistance. J Clin Oncol 2009; 27: 3642-3649.

28. Ng K, Hillmer A, Chuah C, Juan W, Ko T, Teo A, et al. A common BIM deletion polymorphism mediates intrinsic resistance and inferior responses to tyrosine kinase inhibitors in cancer. Nat Med 2012; 18(4): $521-$ 528.

29. de Lavallade H, Khorashad JS, Davis HP, Milojkovic D, Kaeda JS, Goldman JM, et al. Interferon-alpha or homoharringtonine as salvage treatment for chronic myeloid leukemia patients who acquire the T315I BCRABL mutation. Blood. 2007; 110(7):2779-80.

30. Hanfstein B, Muller MC, Kreil S, Ernst T, Schenk T, Lorentz C, et al. Dynamics of mutant BCR-ABL-positive clones after cessation of tyrosine kinase inhibitor therapy. Haematologica. 2011; 96(3):360-6.

31. Rudzki J, Wolf D. Dose Escalation of Imatinib in Chronic-phase Chronic Myeloid Leukemia Patients. Is it Still Reasonable? Expert Rev Hematol 2011; 4(2):153-159.

32. Laneuville P, Dilea C, Yin OQ, Woodman RC, Mestan J, Manley PW. Comparative in vitro cellular data alone are insufficient to predict clinical responses and guide the choice of BCR-ABL inhibitor for treating imatinibresistant chronic myeloid leukemia. J Clin Oncol 2010; 28(11), e169-e171.

33. Yamamoto M, Kurosu T, Kakihana K, Mizuchi D, Miura O. The two major imatinib resistance mutations E255K and T315I enhance the activity of BCR/ABL fusion kinase Biochem Biosphys Res Commun 2004; 319:1272-5.

34. Ernst T, Hoffmann J, Erben P, Hanfstein B, Leitner A, Hehlmann R, et al. ABL single nucleotide polymorphisms may masquerade as BCR-ABL mutations associated with resistance to tyrosine kinase inhibitors in patients with chronic myeloid leukemia. Haematologica 2008; 93(9):1389-1393

35. Nicolini FE, Hayette S, Corm S, Bachy E, Bories D, Tulliez M, et al. Clinical outcome of 27 imatinib mesylateresistant chronic myelogenous leukemia patients harboring a T315I BCR-ABL mutation. Haematologica 2007; 92(09):1238-1241.

36. Hiwase DK, Yeung DT, White DL. Optimizing the Selection of Kinase Inhibitors for Chronic Myeloid Leukemia PatientsExpert Rev Hematol. 2011;4(3):285-299.

37. Talpaz M, Shah NP, Kantarjian H, Donato N, Nicoll J, Paquette R, et al. Dasatinib in imatinib-resistant Philadelphia chromosome-positive leukemias. N Engl J Med. 2006 Jun 15; 354(24):2531-2541.

38. Kantarjian H, Giles F, Wunderle L, Bhalla K, O'Brien S, Wassmann B, et al. Nilotinib in imatinib-resistant CML and Philadelphia chromosome-positive ALL. N Engl J Med. 2006; 354(24):2542-2551.

39. Nicolini FE, Mauro MJ, Martinelli G, Kim D-W, Soverini S, Müller MC, et al. Epidemiologic study on survival of chronic myeloid leukemia and $\mathrm{Ph}^{+}$acute lymphoblastic leukemia patients with BCR-ABLT315I mutation. Blood 2009 ; 114: 5271-5278. 
40. Griswold IJ, MacPartlin M, Bumm T, Goss VL, O’Hare T, Lee KA, et al. Kinase domain mutants of Bcr-Abl exhibit altered transformation potency, kinase activity, and substrate utilization, irrespective of sensitivity to imatinib. Mol Cell Biol. 2006;26:6082-6093.

41. Nicolini FE, Ibrahim AR, Soverini S, Martinelli G, Müller MC, Hochhaus A, et al. The BCR-ABLT315I mutation compromises survival in chronic phase chronic myelogenous leukemia patients resistant to tyrosine kinase inhibitors, in a matched pair analysis. Haematologica 2013; 98(10): 1510-1516.

42. O'Hare T, Walters DK, Stoffregen EP, Jia T, Manley PW, Mestan J, et al. In vitro activity of Bcr-Abl inhibitors AMN107 and BMS-354825 against clinically relevant imatinib-resistant Abl kinase domain mutants. Cancer Res 2005; 65: 4500-4505.

43. Willis SG, Lange T, Demehri S, Otto S, Crossman L, Niederwieser D, et al. High sensitivity detection of BCRABL kinase domain mutations in imatinib-naïve patients: correlation with clonal cytogenetic evolution but not response to therapy. Blood. 2005;106(6):2128-37. 